V Congresso Brasileiro de Informática na Educação (CBIE 2016)

Anais do XXII Workshop de Informática na Escola (WIE 2016)

\title{
Dinâmicas com App Inventor no Apoio ao Aprendizado e no Ensino de Programação
}

\author{
Juliana P. Ribeiro', Marina A. Manso', Marcos A.F. Borges ${ }^{1}$ \\ ${ }^{1}$ Faculdade de Tecnologia - Universidade Estadual de Campinas (UNICAMP) \\ Caixa Postal 456 - Limeira - SP - Brasil
}

\{julianaribeiro@live.com, marinamanso@gmail.com, marcosborges@ft.unicamp.br\}

\begin{abstract}
App Inventor is a free application for creating other applications for Android devices. The App Inventor tool has no specific programming language, using instructions of blocks that can be incorporated in a logical way for the development of applications. This paper discusses ways to encourage the use of App Inventor as a teaching tool for programming and support school learning, evaluating it in several respects. The studies propose a didactic and dynamic way to understand when using the tool and introduce students to the first steps of programming. The results of this project are the construction of the dynamics and assessment of the potential advantages and benefits that this dynamic can bring to student learning.
\end{abstract}

\section{Resumo}

O App Inventor é um aplicativo gratuito para a criação de outros aplicativos (apps) para dispositivos Android. A ferramenta App inventor não possui uma linguagem de programação específica, utilizando-se de blocos de instruções que podem ser encaixados de forma lógica para o desenvolvimento da aplicação. Este trabalho discute formas de incentivar o uso do App Inventor como ferramenta de ensino de programação e apoio ao aprendizado escolar, avaliando-o em vários aspectos. É proposto um método para utilizar a ferramenta e introduzir os alunos aos primeiros passos da programação. Os resultados deste projeto são as dinâmicas e a avaliação das vantagens potenciais e dos benefícios que essas dinâmicas podem trazer ao aprendizado dos alunos.

\section{Palavras- chave}

Lógica de programação, aprendizado, ferramenta App Inventor.

\section{Introdução}

Os estudantes de hoje experimentam a tecnologia de uma forma muito intensiva, através de dispositivos portáteis, telefones e tablets. O que se aprende em sala de aula pode ser complementado facilmente na Internet. A tecnologia pode vir a ser uma extensão da sala de aula na busca por mais conhecimento, viabilizando novos modos de aprendizagem e ensino [Parcianello, Konzen, 2016]. Cabe ao educador usufruir dessa tecnologia como forma de apoio ao ensino em sala de aula. 
O estudo do uso de dispositivos móveis para o aprendizado, principalmente os aparelhos celulares, se justifica pela quantidade de usuários de aparelhos celulares no país, podendo ser considerada uma ferramenta de inclusão digital [Silva, Consolo, 2007]. Conduzir o ensino por meio de projetos reais a serem desenvolvidos pelos próprios estudantes pode ser uma forma de estimular um maior engajamento [Astolfi, Junior, 2016]. Gomes e Melo [2013] apresentam uma abordagem de uso do App Inventor para propiciar experiências mais atrativas de ensino e aprendizagem. Ribeiro [2015] apresenta uma experiência de uso do App Inventor para ensino de lógica de programação que se mostrou mais atrativa, participativa e engajadora, oportunizando a experimentação, a descoberta e o teste. Este trabalho apresenta uma proposta de dinâmicas baseadas em App Inventor, com o uso de dispositivos móveis. Essa proposta foi aplicada a um grupo de jovens do nível médio, e envolve o uso e o desenvolvimento de aplicativos (Apps) para plataforma Android no processo de aprendizado.

Será apresentada na Seção 2 a ferramenta utilizada na pesquisa. Na Seção 3, as dinâmicas propostas, seguida da Seção 4 que apresenta os aplicativos desenvolvidos na pesquisa e da Seção 5 que mostra como as dinâmicas foram aplicadas. Os resultados obtidos através das dinâmicas e a conclusão desse trabalho serão apresentados nas Seções 6 e 7, respectivamente.

\section{A Ferramenta}

Desenvolvida pelo professor Hal Abelson em parceria com uma equipe da Google Education, o projeto do App Inventor é liderado hoje pela equipe do MIT [Massachusetts Institute of Technology, 2016].

App Inventor é uma ferramenta de programação baseada em blocos que permite que usuários, mesmo que iniciantes em programação ou usuários comuns, possam desenvolver aplicativos totalmente funcionais para a plataforma de dispositivos móveis Android [MIT App Inventor, 2016]. App Inventor é simples e de fácil acesso [Massachusetts Institute Of Technology, 2016]. App Inventor permite desenvolver aplicativos para telefones Android usando um navegador da web e um telefone ou emulador conectado. Os servidores do App Inventor guardam o trabalho desenvolvido e ajudam o usuário a manter o controle de seus projetos $\mathrm{O}$ App Inventor é dividido em dois módulos: o App Inventor Designer e o App Inventor Editor de Blocos.

No App Inventor Designer é desenvolvida a interface do aplicativo, com seus componentes escolhidos na "Palette" (em português, palheta) apresentada na Figura 1. $\mathrm{Na}$ palheta ficam todos os componentes utilizáveis num aplicativo. Esta palheta é dividida em seções para facilitar a localização dos componentes, que contêm os componentes básicos como botões, rótulos, imagens, entre outros. Para utilizar um desses componentes basta clicar sobre ele e arrastar para cima da segunda coluna, chamada "Viewer" (em português, visualizador). 
V Congresso Brasileiro de Informática na Educação (CBIE 2016)

Anais do XXII Workshop de Informática na Escola (WIE 2016)

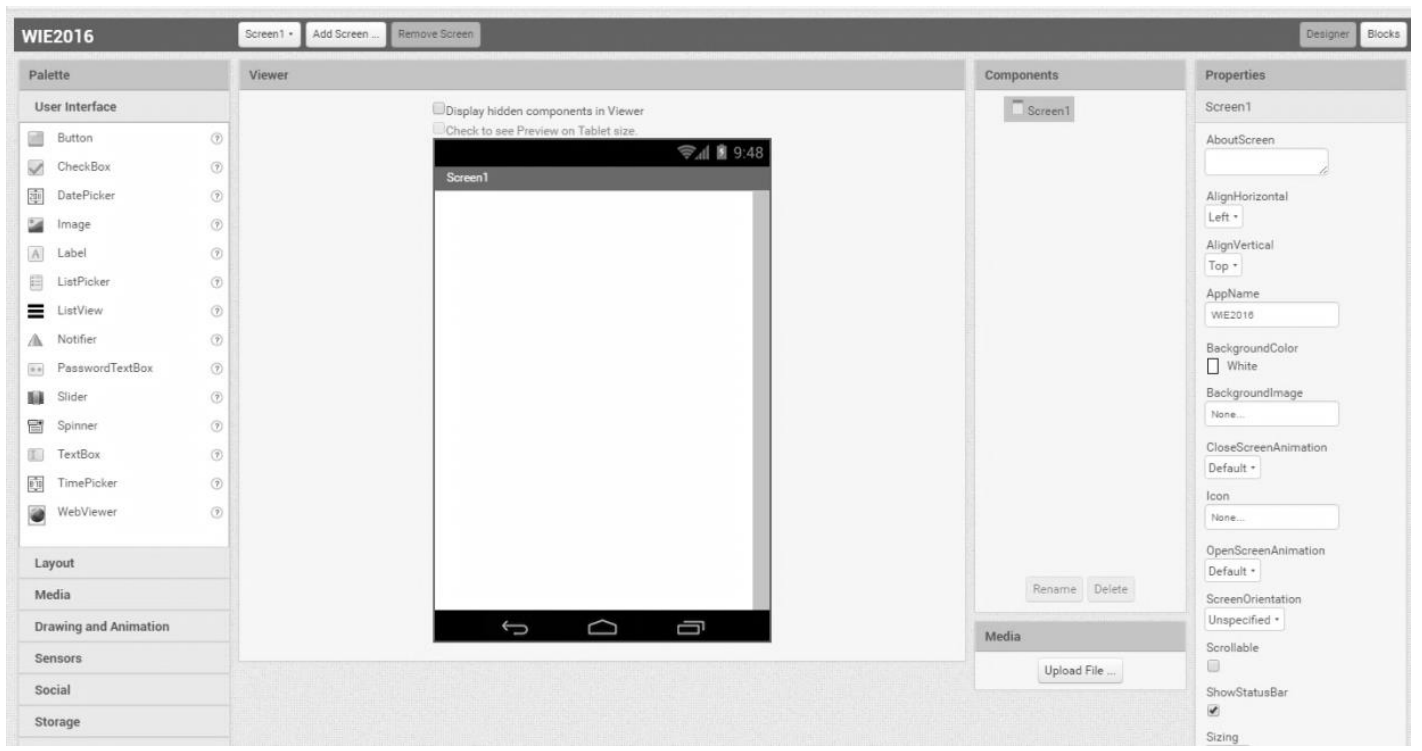

Figura 1: App Inventor Designer

O App Inventor Editor de Blocos é o módulo no qual são adicionados os eventos e funções associados a cada componente predefinido na interface (Figura 2). Isso é feito através de blocos de instruções que são encaixados, especificando como os componentes devem se comportar. Dentro desses blocos de instruções estão, por exemplo, os comandos de repetição, condicionais, variáveis que podem ser editadas, entre outras funcionalidades, muito utilizadas por qualquer aluno de programação. $\mathrm{O}$ usuário apenas tem que montar esses blocos de forma lógica, para que sua aplicação realize as atividades esperadas.

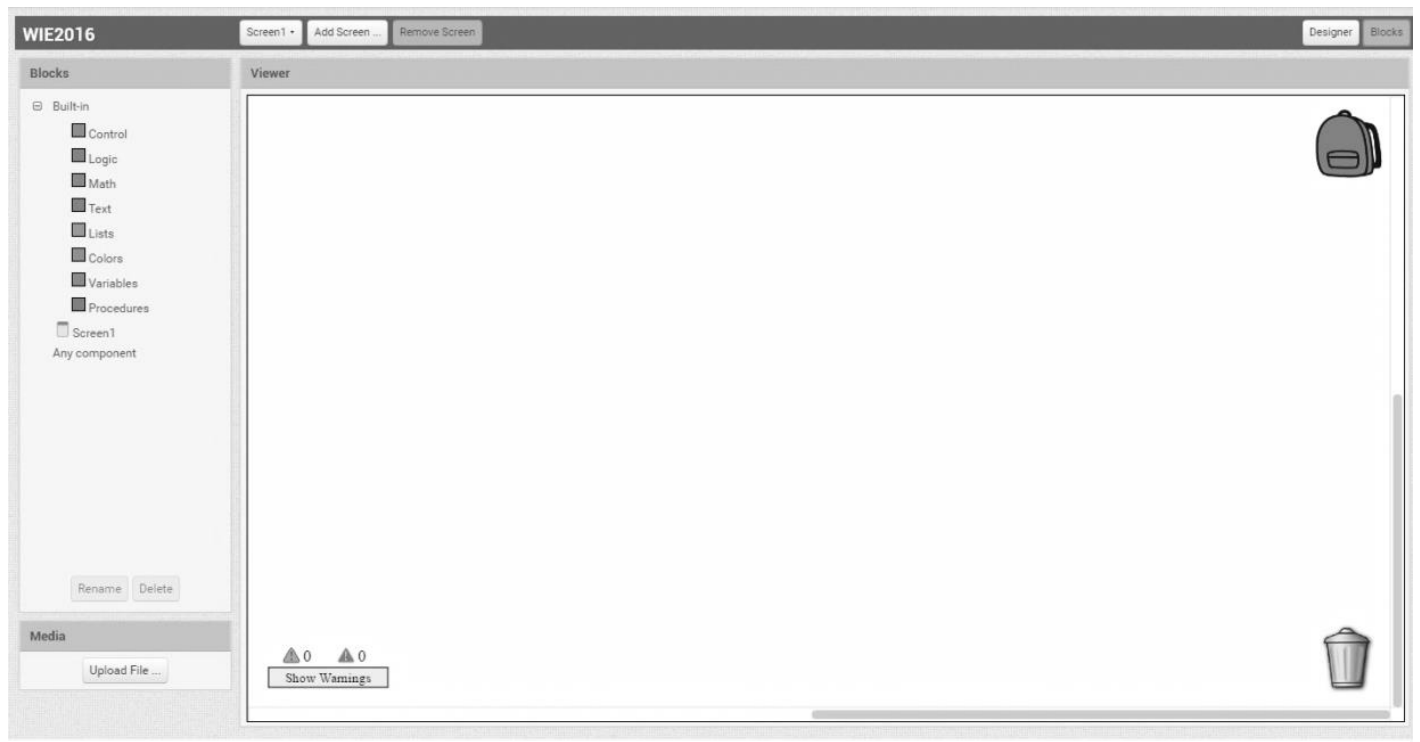

Figura 2: App Inventor Editor de Blocos

\section{Proposta de dinâmica}

Este projeto teve como objetivo propor dinâmicas de aplicação de App Inventor e analisar o impacto da integração da lógica de programação para apoio ao aprendizado. 
V Congresso Brasileiro de Informática na Educação (CBIE 2016)

Anais do XXII Workshop de Informática na Escola (WIE 2016)

Foram conduzidas dinâmicas de desenvolvimento de aplicativos móveis, baseadas em App Inventor, com uma classe de jovens de ensino médio, de quatorze a dezessete anos de idade, durante duas aulas, de quatro horas cada, em uma turma de 40 alunos.

As dinâmicas propostas têm a seguinte estrutura: inicialmente, os jovens são instruídos a sentar-se na frente do computador, sendo informados que eles não serão avaliados com relação ao seu desempenho, e que não há obrigatoriedade nenhuma na participação: no experimento relatado neste trabalho, não houve desistências. Os participantes também são motivados a perguntar caso haja alguma dúvida. Em seguida, são apresentados alguns aplicativos prontos, para servirem como exemplo de aplicações: os participantes têm alguns minutos para interagir com aplicativos, sendo questionados ao longo dessa experimentação se acham que são capazes de fazerem algo similar. Em seguida, é feita uma explicação sobre o que é o App Inventor e são apresentados os seus componentes e sua interface. Apresenta-se, então, a interface de programação, na qual se explica o que é programação em blocos, e que eles devem montar um"quebra-cabeças" para chegar ao resultado desejado. O próximo passo é o desenvolvimento de aplicativos, conforme descrito na próxima seção. Ao final da dinâmica, os jovens devem responder, de forma anônima para evitar constrangimentos, o questionário apresentado na Quadro 1, para que se análise os resultados obtidos.

\begin{tabular}{|l|l|l|}
\hline $\begin{array}{l}|l| \\
\text { 1- Você achou que criar aplicativos é: }\end{array}$ & [ ] Não \\
\hline [ ] Fácil & [ ] Médio & [ ] Difícil \\
\hline 2-Você teve dificuldade ao criar aplicativos. Se sim, qual? \\
\hline []Sim R: & [ ] Não \\
\hline 3- Você usa smartphones com frequência?
\end{tabular}

Quadro 1: Questionário de Avaliação

\section{Aplicativos Desenvolvidos}

Foram desenvolvidos vários aplicativos para a demonstração da ferramenta, motivando os jovens previamente à aplicação da dinâmica. Outros aplicativos foram propostos para serem construídos durante a condução dessas dinâmicas.

O primeiro aplicativo criado é um aplicativo de comparação de números, visando o aprendizado básico da lógica de comparação do App Inventor e uma apresentação inicial da plataforma. O Aplicativo resume-se em criar dois números escolhidos aleatoriamente (randomicamente) em ambos os lados e a criação de dois botões de 
V Congresso Brasileiro de Informática na Educação (CBIE 2016)

Anais do XXII Workshop de Informática na Escola (WIE 2016)

comparação com o sinal de maior e menor. Caso o participante faça a escolha correta, é exibida uma mensagem parabenizando-o e, caso contrário, é apresentada uma mensagem de incentivo para tentar novamente (Figura 3).

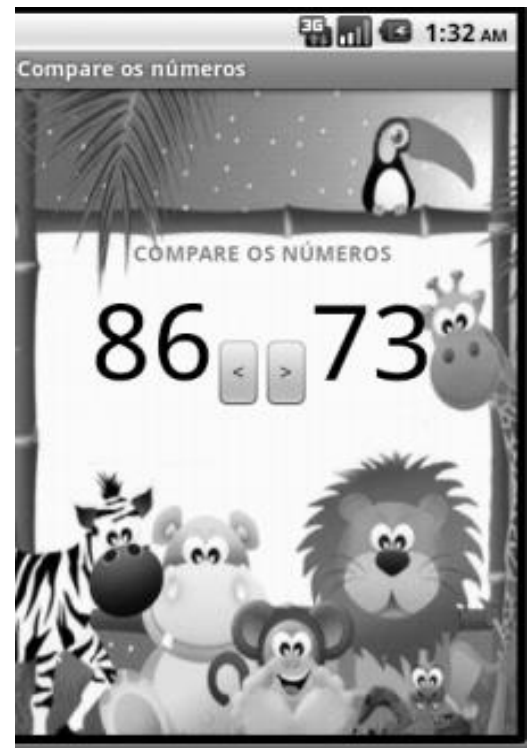

Figura 3: App Compara Números

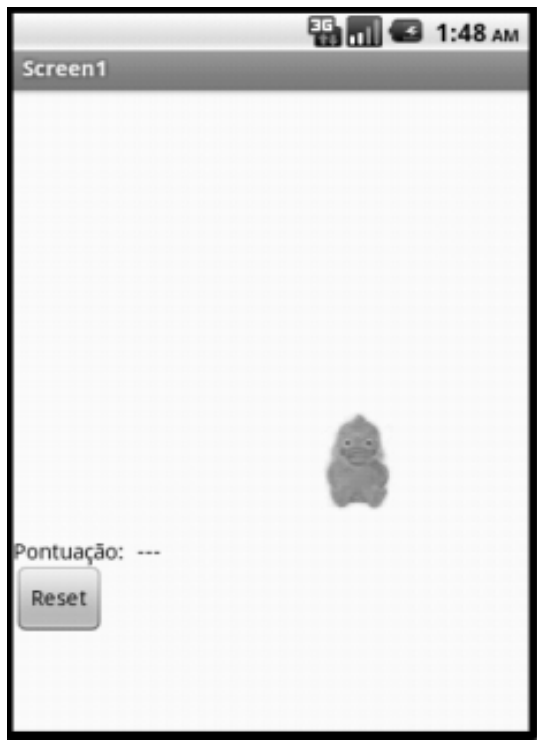

Figura 4: Jogo do Pato Maluco

O segundo aplicativo desenvolvido foi o "Jogo do Pato Maluco" (foi apelidado assim pelos participantes que fizeram a primeira dinâmica). O jogo consiste em "caçar" um pato, ou qualquer outro animal em que os jovens importassem a foto, clicando no celular no local exato em que o pato estava. Entretanto, a mudança de localização do pato era imprevisível e frequente. Para a construção desse jogo, os participantes aprenderam conceitos introdutórios de lógica da programação e ainda tiveram que treinar os seus conhecimentos de matemática, em planos cartesianos, pois deveriam calcular os pontos em que o pato poderia aparecer na tela (Figura 4).

O terceiro aplicativo desenvolvido, novamente em forma de jogo, utilizava os pontos cardeais, construindo assim uma espécie de bússola. Utilizando o sensor de movimento do celular e a interface de conexão oferecida pelo App Inventor, pode-se adquirir a informação para onde o celular está sendo apontado. Com base nisso, foi desenvolvido um jogo onde o usuário aponta o celular para um dos pontos cardeais indicados (Norte, Sul, Leste ou Oeste) e uma porta se abre, passando assim para a próxima fase (Figura 5). 
V Congresso Brasileiro de Informática na Educação (CBIE 2016)

Anais do XXII Workshop de Informática na Escola (WIE 2016)

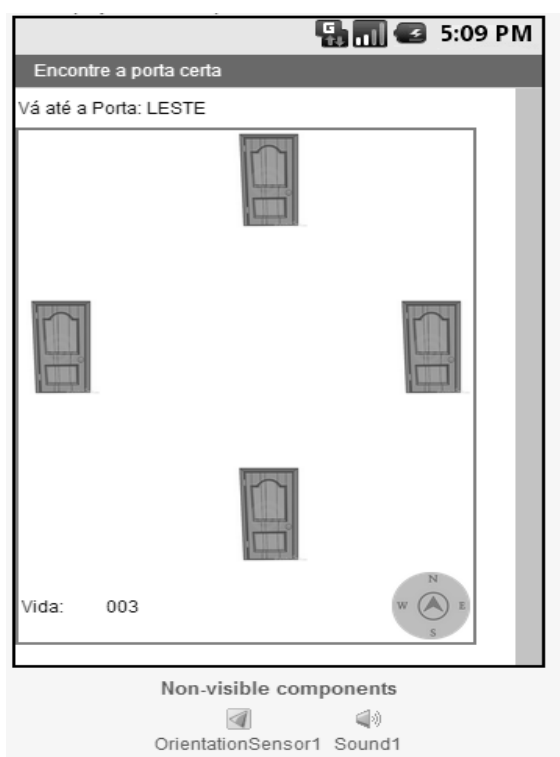

Figura 5: App Pontos Cardeais

Foram desenvolvidos também um aplicativo que emitia sons ao pressionar um botão, um aplicativo para anotações no celular, outro aplicativo para exibição de mapas, aplicativos para a cotação de dólar entre outros, que ilustram a capacidade do App Inventor como ferramenta de desenvolvimento.

Outro aplicativo desenvolvido integra o App Inventor ao LEGO Mindstorm [The Lego Group, 2016]. Com esse aplicativo, os jovens são capazes de controlar um robô com o seu celular. Este aplicativo utiliza, além dos componentes da palheta do App Inventor, a palheta integrada da Lego. Lançada no ano de 1998, o sistema LEGO Mindstorms é uma linha específica de LEGO programável para a área de educação tecnológica. Sua utilização baseia-se no aprendizado lúdico. Seu desenvolvimento se deu graças à parceria entre o LEGO Group e o Media Lab do MIT [Massaschusetts Institute of Technology, 2016]. Com este sistema é possível projetar um Robô com funções pré-programadas.

O App Inventor vem com uma biblioteca para interação com o Lego Mindstorm. Com essa biblioteca, é possível programar os sensores na plataforma e fazer a integração do robô com o celular através de conexão bluetooth, podendo controlar o robô conforme foi programado.

Para utilizar os aplicativos nas dinâmicas, os robôs de LEGO devem estar previamente construídos (Figura 6). Embora a montagem seja fácil e lúdica, não faz parte do objetivo, e prolongaria demais as dinâmicas. Para o aplicativo de demonstração foi feito um controle para os motores de movimento, fazendo com que o robô se deslocasse na direção desejada pelos participantes (Figura 7). 
V Congresso Brasileiro de Informática na Educação (CBIE 2016)

Anais do XXII Workshop de Informática na Escola (WIE 2016)

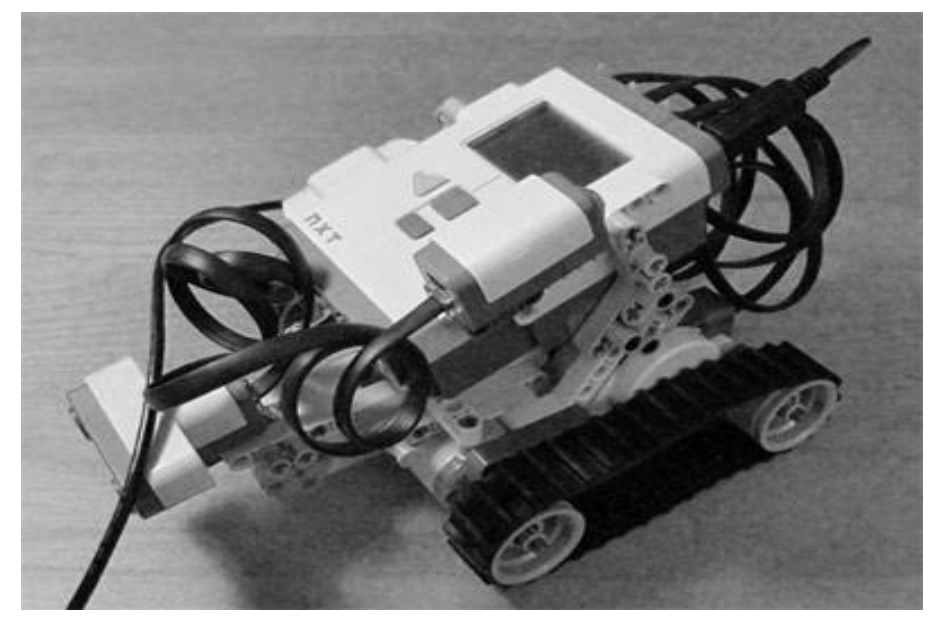

Figura 6: Robô feito de LEGO Mindstorm e aplicativo de controle

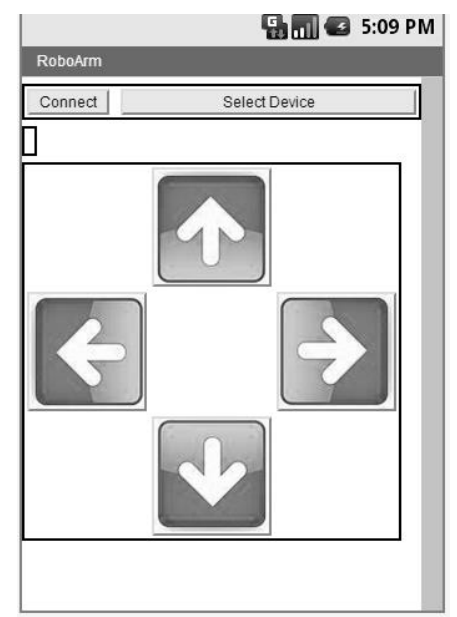

Figura 7: Controle do robô

\section{Aplicação das dinâmicas}

Um primeiro conjunto de dinâmicas foi conduzido para avaliar as propostas. Os participantes foram divididos por idade, e, de acordo com a idade, foi designada uma tarefa para cada grupo. Os alunos são acompanhados passo a passo e receberam ajuda sempre que não sabiam como prosseguir.

Foi observado o desempenho dos participantes durante a dinâmica, bem como seu interesse pelo assunto durante o desenvolvimento das dinâmicas, discutidos na Seção 6.

\section{Resultados}

O desempenho geral dos participantes durante as dinâmicas foi satisfatório. Os grupos conseguiram evoluir muito bem, e no decorrer do processo, surgiram várias dúvidas, que foram esclarecidas sem grandes transtornos. Os aplicativos finais ficaram com o resultado esperado e os participantes ficaram entusiasmados com a ideia de testar os aplicativos em seus próprios celulares. 


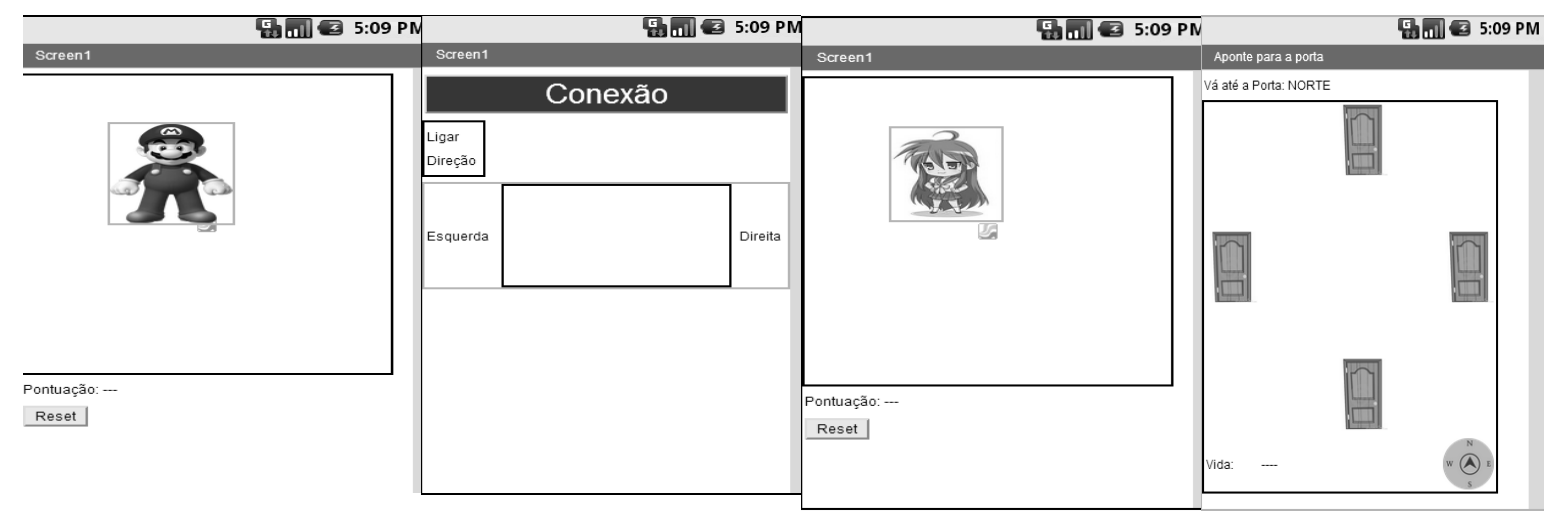

Figura 7: Resultados de alguns apps desenvolvidos pelos participantes

Ao final da dinâmica, foi perguntado aos participantes o que tinha mudado sobre a sua visão de programação e criação de aplicativos. Um dos participantes, respondeu: "Eu pensava que programação era algo extremamente complicado, só para as pessoas realmente inteligentes e não para mim, mas depois de entender como funciona essa lógica, eu consigo imaginar diversas formas de uso para poder criar outros aplicativos. Controlar o robô foi uma experiência muito divertida, ver como funciona e logo em seguida ver funcionando, me fez pensar e ver novas possibilidades, quem sabe eu não vire um programador agora né?".

Foram elaborados dois gráficos com base nos resultados do questionário proposto aos participantes. O primeiro gráfico (Gráfico 1) ilustra a primeira pergunta sobre a facilidade de criação de aplicativos com App Inventor e o segundo gráfico (Gráfico 2) apresenta a tabulação da última pergunta sobre a motivação dos alunos a continuarem aprendendo programação.

Como vemos no Gráfico 1, 79\% dos participantes acharam fácil a criação de aplicativos depois das dinâmicas propostas, contrapondo a dificuldade em programação que eles julgavam ter antes do início das atividades, pois comparando com os dados da terceira pergunta, $84 \%$ dos participantes achavam que não eram capazes de fazer as atividades antes de começarem as dinâmicas.

Os alunos também saíram bem motivados. Mesmo com $20 \%$ achando a atividade média ou difícil, conforme o Gráfico 2, apenas $4 \%$ não continuariam tentando, o que mostra que $96 \%$ saíram motivados a tentar, mesmo tendo algumas dificuldades.

Com base na pergunta 2 , observou-se que entre os alunos que indicaram ter dificuldade, $67 \%$ encontraram dificuldade no inglês, dado que todos os comandos da plataforma e sua interface na versão utilizada, eram em inglês. Foi verificado que a dificuldade dobra, se o usuário não tem um nível no mínimo básico da língua. Essa dificuldade apresentada com a língua inglesa poderia inviabilizar a apresentação da plataforma para alunos mais novos, nas quais, a dificuldade com a língua inglesa é 
V Congresso Brasileiro de Informática na Educação (CBIE 2016)

Anais do XXII Workshop de Informática na Escola (WIE 2016)

maior. No entanto, essa dificuldade não é mais relevante, visto que foi disponibilizada uma versão em português do App Inventor em 2016 [VALLE, 2016].

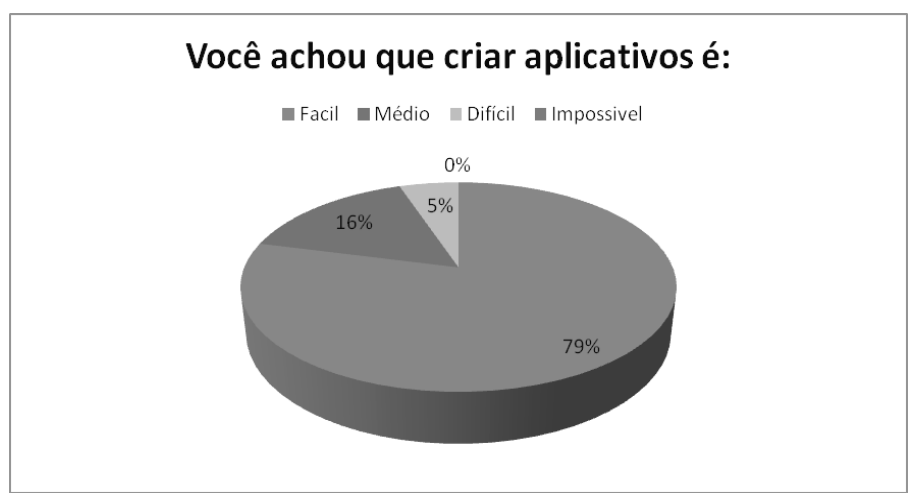

Gráfico 1: Facilidade na criação de Apps

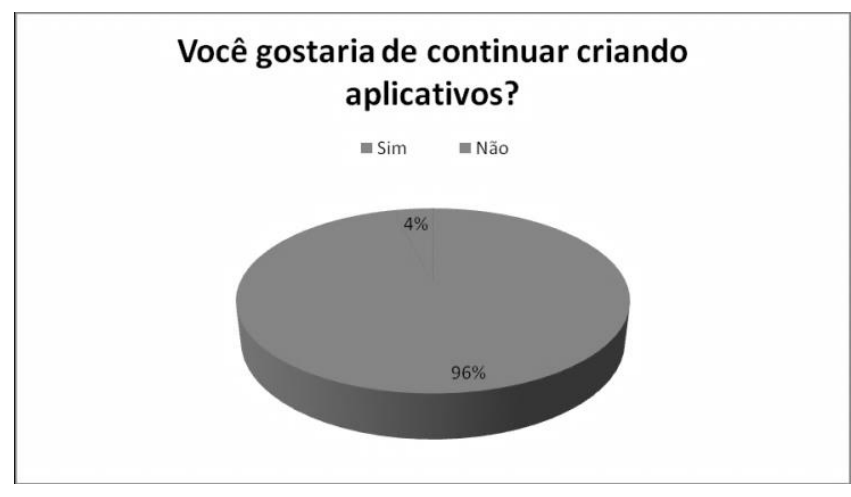

Gráfico 2: Interesse pela criação de aplicativos futuramente

\section{Conclusão}

Este trabalho teve como objetivo propor uma metodologia para apoio ao ensino direcionada a jovens do nível médio com base na ferramenta interativa App Inventor, que permite a programação para dispositivos móveis de forma facilitada e dinâmica. Foram construídos vários aplicativos para testes e propostas dinâmicas de desenvolvimento de aplicativos móveis. As dinâmicas foram aplicadas com um grupo de jovens de ensino médio, de forma a analisar a integração da lógica de programação no apoio ao aprendizado.

As dinâmicas foram conduzidas e um questionário respondido pelos participantes. Com os dados dos questionários, pode-se concluir que o App Inventor é uma plataforma que oferece oportunidades para uso em processos de aprendizado, dando ao mesmo tempo para os participantes, uma nova visão sobre programação e a carreira na área de computação.

Como trabalho futuro, seria interessante refazer as dinâmicas com a nova versão do App Inventor, em Português, para verificar qual a diferença que essa questão traria nos resultados.

\section{Referências Bibliográficas}

Astolfi, G.; Junior, D.L.(2016). "Ensino de Linguagem de Programação com ênfase na Aprendizagem Significativa." XXXVI Congresso da Sociedade Brasileira de Computação - WEI- 24 Workshop sobre Educação em Computação. 
V Congresso Brasileiro de Informática na Educação (CBIE 2016)

Anais do XXII Workshop de Informática na Escola (WIE 2016)

Gomes,T.C.S.;Melo, J.C.B. (2013). “App Inventor for Android: Uma nova possibilidade para o ensino de lógica de programação." II Congresso Brasileiro de Informática na Educação (CBIE 2013) - Workshops (WCBIE 2013).

Massachusetts Institute of Technology (2016). Disponível em:

< http://web.mit.edu/ > Acessado em 11/03/2016.

MIT App Inventor(2016). Disponível em: <http://appinventor.mit.edu/explore/> Acessado em 08/03/2016.

Parcianello, L; Konzen, P.C (2016). "Docência no ensino superior: o uso das novas tecnologias na formação de professores na licenciatura". Disponível em: $<\mathrm{http}$ ://www.arcos.org.br/artigos/docencia-no-ensino-superior-o-uso-das-novastecnologias-na-formacao-de-professores-na-licenciatura/\#topo> Acessado em 08/03/2016.

Ribeiro,I.(2015). "Redesigning traditional courses: Use of technology in the classroom." ICERI2015:8TH International Conference Of Education, Research and Innovation.

Silva, M.G.M; Consolo, A.T (2007). "Uso de dispositivos móveis na educação - o SMS como auxiliar na mediação pedagógica de cursos a distância”. Disponível em:〈http://www.5e.com.br/infodesign/146/Dispositivos_moveis.pdf>. Acessado em $10 / 03 / 2016$.

The Lego Group (2016) - Lego Mindstorms. Disponível em: <http://www.lego.com/enus/mindstorms/?domainredir=mindstorms.lego.com>. Acessado em 11/03/2016.

Valle, E. (2016) - "MIT App Inventor Translated to Brazilian Portuguese"- Disponível em < http://appinventor.mit.edu/explore/blogs/josh/2016/01/mit-0.html>. Acessado em 09/08/2016. 